CASE REPORT

\title{
Congenital dermatofibrosarcoma protuberans with fibrosarcomatous and myxoid change
}

\author{
W Gu, A Ogose, H Kawashima, H Umezu, N Kudo, T Hotta, N Endo
}

J Clin Pathol 2005;58:984-986. doi: 10.1136/icp.2004.024430

This report describes a case of congenital dermatofibrosarcoma protuberans (DFSP) with fibrosarcomatous (FS) and myxoid areas. Immunohistochemical results showed that tumour cells in ordinary DFSP areas were diffusely positive for CD34, whereas in the FS and myxoid areas, few tumour cells were positive for this antigen. $\mathrm{Ki}-67$ positive tumour cell numbers were greater in the FS (11.8\%) and myxoid areas $(19.8 \%)$ relative to ordinary DFSP areas $(2.2 \%)$. Reverse transcription polymerase chain reaction and sequence analysis showed the presence of an identical COL1A1PDGFB fusion transcript in ordinary DFSP (plaque-like area), FS, and myxoid areas of DFSP. These results indicate that the three components of DFSP have a common histogenesis. This study documents the first application of gene analysis involving the myxoid area of DFSP.

A 29 year old man presented with a firm, congenital blue/ black plaque on the upper aspect of the left pubis apparent since birth. The lesion was thought to be a birthmark and had not been subjected to further scrutiny. However, the lesion slowly enlarged and at 27 years of age, two protuberant tumours appeared on the surface of the initial plaque and grew rapidly. Two years later, the two tumours had a diameter of $5 \mathrm{~cm}$; moreover, one of the tumours became ulcerated and haemorrhaged (fig 1). An incisional biopsy revealed dermatofibrosarcoma protuberans (DFSP). Tumour resection was performed followed by coverage with the rectus abdominis myocutaneous flap. The patient shows no evidence of recurrence 12 months after surgery.

\section{Immunohistochemical and cytogenetic results}

The histological features of the tumour showed three distinct components. The brownish, plaque-like lesion (fig lA) was comprised of ordinary DFSP. The slender, fibroblastic tumour cells, which proliferated into the deep dermis, showed a monotonous storiform pattern (fig $\mathrm{lAl}$ ). Little nuclear pleomorphism and slight mitotic activity were apparent. Immunohistochemically, the tumour cells were diffusely positive for CD34 (fig 1A2). The Ki-67 index was $2.2 \%$ in the ordinary DFSP area (fig 1A3). The nodular tumour at the right side (fig 1B) was composed of fibrosarcomatous elements. This area consisted of dense fascicles of cells demonstrating mild nuclear pleomorphism, which displayed a herringbone pattern (fig 1B1). A few tumour cells were positive for CD34 (fig 1B2). The Ki-67 index was $11.8 \%$ (fig 1B3). The ulcerated large nodule and small nodules at the left side (fig 1C, $C^{\prime}$ ) were characterised by myxoid features. The tumour cells exhibited moderate nuclear pleomorphism with prominent vascular proliferation (fig 1C1). A few tumour cells were positive for CD34 (fig 1C2). The Ki-67 index was 19.8\% (fig lC3). Tumour cells in all components were negative for epithelial membrane antigen, CAM 5.2, AEl/AE3, CD31, desmin, and S100 protein.

Cytogenetic analysis revealed the presence of a supernumerary ring chromosome after a five day culture; the karyotype was $49, \mathrm{XY},+18,+\mathrm{rl}$ (fig 2A). The COLlAl-PDGFB (encoding collagen type $1 \alpha 1$-platelet derived growth factor $\mathrm{B}$ chain) fusion transcript (370 bp) was detected by amplification with primers COLIAl exon 40 and PDGFB exon 2 in the ordinary (plaque-like), FS, and myxoid areas of DFSP (fig 2B). This result suggested that the identical fusion transcript existed in the three distinct areas of DFSP. To identify the breakpoint, nucleotide sequence analysis was performed using the $370 \mathrm{bp}$ polymerase chain reaction product. Sequence analysis demonstrated that the end of exon 42 in the COLlAl gene was fused with the start of exon 2 in the PDGFB gene.

\section{DISCUSSION}

To the best of our knowledge, 27 cases of congenital DFSP have been documented. ${ }^{1}$ The diagnosis of congenital DFSP in infancy or childhood remains difficult clinically because the lesion often resembles a vascular birthmark, especially in its early stages. Conventional DFSPs including myxoid variants rarely metastasise and cause death, whereas the fibrosarcomatous variant has an approximately $15 \%$ risk of metastasis and a $5.8 \%$ risk of tumour related death. ${ }^{2}$

"This is the first report of the detection of fusion gene expression in the myxoid area of dermatofibrosarcoma protuberans"

Immunohistochemically, DFSP is characterised by the relatively consistent presence of CD34. ${ }^{3}$ In our present case, tumour cells in ordinary DFSP areas were diffusely positive for CD34, whereas in DFSP-FS and myxoid areas, only a few tumour cells were positive for this marker. Interestingly, Ki67 positive tumour cell numbers were greater in DFSP-FS and myxoid areas relative to ordinary DFSP. These results suggested that DFSP-FS and myxoid areas might have a tendency towards reduced or absent CD34 immunopositivity while maintaining qualities associated with tumour progression or rapid proliferation. Supernumerary ring chromosomes containing amplified sequences from chromosomes 17 and 22 are the most frequent abnormalities seen in DFSP. ${ }^{4}$ Sirvent

Abbreviations: DFSP, epithelial membrane antigen; DFSP-FS, dermatofibrosarcoma protuberans with fibrosarcomatous area; FS, fibrosarcomatous 

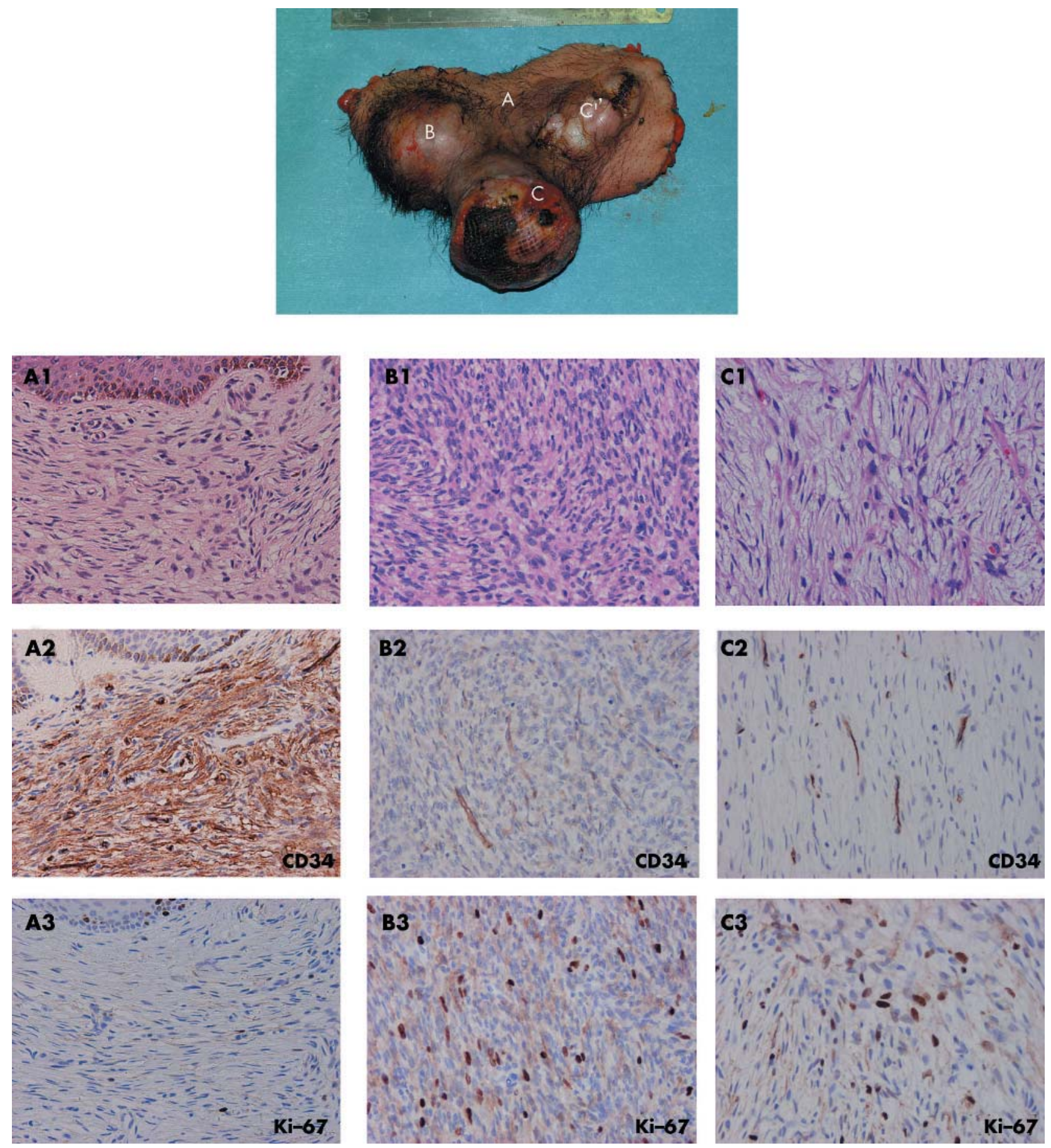

Figure 1 Top panel: (A) ordinary (plaque-like) area of dermatofibrosarcoma protuberans (DFSP); (B) fibrosarcomatous area of DFSP; (C, $C^{\prime}$ ), myxoid area of DFSP. (A1-C1) Haematoxylin and eosin staining, (A2-C2) CD34 immunohistochemical staining, and (A3-C3) Ki-67 immunohistochemical staining were performed for the three different components of DFSP, respectively.

and colleagues ${ }^{5}$ reported the presence of ring chromosomes in most adult DFSP cases; in contrast, all childhood cases displayed translocation derivatives. In our present case, a supernumerary ring chromosome was identified by chromosomal analysis. In the FS area, the ring chromosomes may represent the final chromosomal outcome of a multistep progression for tumours. Patient age might affect the expression form of the fusion gene. Multiplex reverse transcription polymerase chain reaction detected COLlAlPDGFB fusion transcripts via amplification with the primers
COLlAl exon 40 and PDGFB exon 2 in all components of DFSP. Sequence results revealed that the end of exon 42 in the COLlAl gene was fused with the start of exon 2 in the PDGFB gene. This fusion gene has been identified in both ordinary DFSP and FS areas of DFSP ${ }^{6}$; however, this is the first report of the detection of fusion gene expression in the myxoid area of DFSP. The coexpression of the identical fusion gene in the three components of DFSP indicates that the three components originated from the same tumour cells, and that a genetic link existed among these tumours. 
A
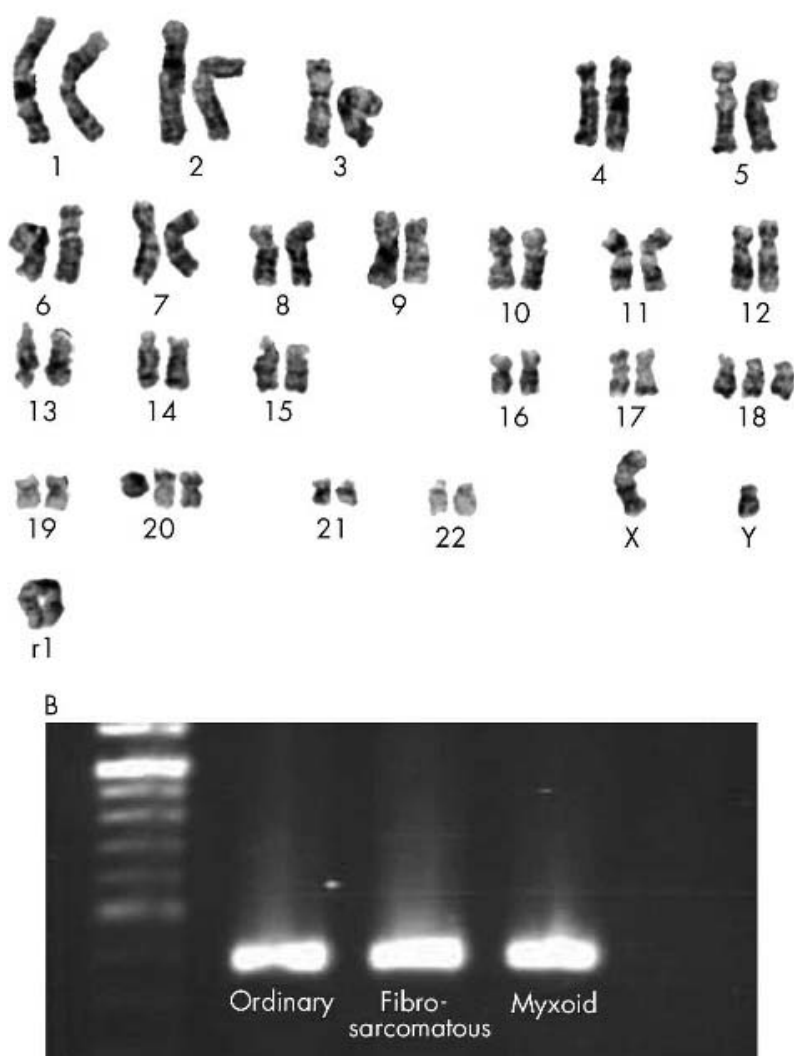

Figure 2 (A) Representative karyotype of this case of dermatofibrosarcoma protuberans (DFSP). $r 1$ is the supernumerary ring chromosome (B) Expression of the COL1A1-PDGFB fusion gene. The fusion transcript with primers COL1A1 exon 40 and PDGFB exon 2 was expressed in all three components of DFSP.

\section{ACKNOWLEDGEMENTS}

This study was supported in part by Niigata Orthopaedic International Exchange Fund.

\section{Authors' affiliations}

W Gu, A Ogose, H Kawashima, N Kudo, T Hotta, N Endo, Division of Orthopaedic Surgery, Niigata University Graduate School of Medical and Dental Sciences, 1-757 Asahimachi-Dori, Niigata, 951-8510 Japan

\section{Take home messages}

- We describe a case of congenital dermatofibrosarcoma protuberans (DFSP) with fibrosarcomatous (FS) and myxoid areas

- Tumour cells in congenital DFSP areas were diffusely positive for CD34, whereas in the DFSP-FS area and myxoid area, only a few tumour cells were positive for this marker

- The three components of DFSP originated from the same tumour cells and a genetic link existed among these tumours

H Umezu, Division of Pathology, Niigata University Hospital, Niigata, Japan

W Gu, Division of Orthopaedic Surgery, First Affiliated Hospital of Harbin Medical University, Harbin, China

The patient gave informed consent for this report to be published.

Correspondence to: Dr A Ogose, Division of Orthopaedic Surgery, Niigata University Graduate School of Medical and Dental Sciences, 1-757 Asahimachi-Dori, Niigata, 951-8510 Japan;

aogose@med.niigata-u.ac.jp

Accepted for publication 15 December 2004

\section{REFERENCES}

1 Weinstein JM, Drolet BA, Esterly NB, et al. Congenital dermatofibrosarcoma protuberans: variability in presentation. Arch Dermatol 2003;139:207-11.

2 Mentzel T, Beham A, Katenkamp D, et al. Fibrosarcomatous ("high-grade") dermatofibrosarcoma protuberans: clinicopathologic and immunohistochemical study of a series of 41 cases with emphasis on prognostic significance. Am J Surg Pathol 1998;22:576-87.

3 Goldblum JR, Tuthill RJ. CD34 and factor-XIlla immunoreactivity in dermatofibrosarcoma protuberans and dermatofibroma. Am J Dermatopathol 1997; 19:147-53.

4 Minoletti F, Miozzo M, Pedeutour F, et al. Involvement of chromosomes 17 and 22 in dermatofibrosarcoma protuberans. Genes Chromosomes Cancer $1995 ; 13: 62-5$

5 Sirvent N, Maire G, Pedeutour F. Genetics of dermatofibrosarcoma protuberans family of tumors: from ring chromosomes to tyrosine kinase inhibitor treatment. Genes Chromosomes Cancer 2003;37:1-19.

6 Wang J, Morimitsu Y, Okamoto S, et al. COL1A1-PDGFB fusion transcripts in fibrosarcomatous areas of six dermatofibrosarcomas protuberans. J Mol Diagn 2000;2:47-52. 